\title{
Whole-exome sequencing gives additional benefits compared to candidate gene sequencing in the molecular diagnosis of children with growth hormone or IGF-1 insensitivity
}

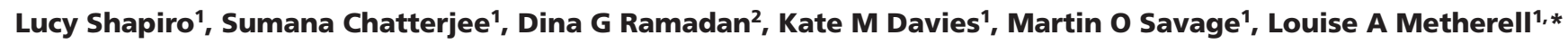
and Helen L Storr'1,*

${ }^{1}$ Centre for Endocrinology, William Harvey Research Institute, Barts and the London School of Medicine and Dentistry, Queen Mary University of London, London, UK and ${ }^{2}$ Department of Pediatrics and Endocrinology Unit, Sabah Hospital, Safat, Kuwait

*(L A Metherell and H L Storr contributed equally to this work)

Correspondence should be addressed to $\mathrm{H}$ Storr

Email

h.l.storr@qmul.ac.uk

\begin{abstract}
Background: GH insensitivity (GHI) is characterised by short stature, IGF-1 deficiency and normal/elevated serum GH. IGF-1 insensitivity results in pre- and post-natal growth failure with normal/high IGF-1 levels. The prevalence of genetic defects is unknown.

Objective: To identify the underlying genetic diagnoses in a paediatric cohort with GH or IGF-1 insensitivity using candidate gene (CGS) and whole-exome sequencing (WES) and assess factors associated with the discovery of a genetic defect.

Methods: We undertook a prospective study of 132 patients with short stature and suspected GH or IGF-1 insensitivity referred to our centre for genetic analysis. 107 (96 GHI, 88 probands; 11 IGF-1 insensitivity, 9 probands) underwent CGS. WES was performed in those with no defined genetic aetiology following CGS.

Results: A genetic diagnosis was discovered 38/107 (36\%) patients (32\% probands) by CGS. WES revealed 11 patients with genetic variants in genes known to cause short stature. A further 2 patients had hypomethylation in the $\mathrm{H} 19 /$ IGF2 region or mUPD7 consistent with Silver-Russell Syndrome (total with genetic diagnosis 51/107, 48\% or 41/97, $42 \%$ probands). WES also identified homozygous putative variants in FANCA and PHKB in 2 patients. Low height SDS and consanguinity were highly predictive for identifying a genetic defect.

Conclusions: Comprehensive genetic testing confirms the genetic heterogeneity of GH/IGF-1 insensitivity and successfully identified the genetic aetiology in a significant proportion of cases. WES is rapid and may isolate genetic variants that have been missed by traditional clinically driven genetic testing. This emphasises the benefits of specialist diagnostic centres.

European Journal of

Endocrinology

(2017) 177, 485-501

\section{Introduction}

Short stature is one of the most common reasons for referral to paediatric endocrinologists. Patients with defects in growth hormone (GH) action or GH insensitivity (GHI) frequently present with severe phenotypes (height

SDS $-\leq 2.5)$ and the aetiology often remains uncertain. Consequently, many patients are classified as having idiopathic short stature (ISS) and pose a significant diagnostic and management challenge.
\end{abstract}

() 2017 European Society of Endocrinology Printed in Great Britain 
The growth hormone-insulin-like growth factor-1 (GH-IGF-1) axis is essential for human growth (1). The cardinal features of GHI are severe growth failure, normal GH secretion and IGF-1 deficiency (IGFD). Monogenic defects leading to GHI have been discovered in $\operatorname{GHR}(2,3)$, STAT5B (4, 5), IGFALS (6), PAPPA2 (7) and IGF1 (8). IGF-1 insensitivity secondary to $I G F 1 R$ gene mutations exists as part of the continuum and leads to a similar phenotype (9). In cases of IGF-1 resistance, the IGF-1 levels are high (10). Depending on the genetic defect, associated clinical and dysmorphic features may be present including midfacial hypoplasia and frontal bossing (GHR, STAT5B) $(4,11)$, immune deficiency (STAT5B) (4), pubertal delay (IGFALS, STAT5B, GHR) $(4,11,12)$, decreased bone mineral density (PAPPA2) (7), developmental delay, microcephaly and in-utero growth retardation (IGF1, IGF1R) (1). 3M, Silver-Russell (SRS) and Noonan (NS) syndromes have phenotypes that can overlap with GHI $(10,13,14)$. 3M syndrome (OMIM 273750) results in pre- and post-natal growth restriction, prominent heels, facial dysmorphism and distinct radiological features (15). The genetics are incompletely understood, but mutations in cullin 7 (CUL7) (70\%), obscurin-like 1 (OBSL1) (25\%) and coiled coil domain-containing 8 (CCDC8) (5\%) genes have been identified $(16,17)$. SRS is characterised by intrauterine and/or post-natal growth retardation and is caused by maternal uniparental disomy of chromosome seven (matUPD7) and hypomethylation of the imprinted H19/ IGF2 domain of chromosome 11p15 in 10 and 35\%-65\% cases, respectively (18). Noonan syndrome results from autosomal dominant mutations in the Ras/mitogenactivated protein kinase signalling pathways (PTPN11, SOS1, SOS2, RAF1, BRAF, NRAS, KRAS, HRAS, CBL, RIT1, RASA2, MAP2K1, MAP2K2, A2ML1 LZTR1 and SHOC2 genes) in $\sim 70 \%$ patients $(19,20)$.

The identification of a pathogenic molecular defect is important for families and clinicians. A genetic diagnosis ends uncertainty, avoids unnecessary investigations and treatment and allows appropriate genetic counselling and the identification of possible co-morbidities in syndromic short stature. A genetic diagnosis may also lead to earlier initiation of therapy and therefore a better long-term treatment response (21).

Genetic defects can be identified by traditional Sanger sequencing of the most likely candidate genes (candidate gene sequencing, CGS) or by next-generation sequencing e.g. whole-exome sequencing (WES). CGS is clinically driven and is reliable when the affected gene can be predicted with a high degree of certainty. Its success depends on the accurate clinical phenotyping of patients and is limited in growth disorders with overlapping, highly variable or subtle features (22). It is also time-consuming and costly if a number of genes are analysed. In contrast, WES allows the simultaneous screening of the entire coding DNA of an individual and is therefore extremely cost-effective if multiple genes are to be investigated.

As a genetic reference centre, we undertook CGS in a cohort of patients with short stature and suspected GH or IGF-1 insensitivity. This is an extension of our previous work and some of the patients have been previously reported (10). WES was completed in patients with no diagnosis following CGS. Our data demonstrate the importance of comprehensive genetic analysis in severe short stature, particularly the utility of WES in securing a molecular diagnosis where CGS has yielded negative results.

\section{Subjects and methods}

\section{Patients}

Between 2008 and 2017, our centre received 132 referrals $(75 \mathrm{M})$ for genetic investigation. Patients were referred from: UK $(n=77)$, Kuwait $(n=20)$, Poland $(n=10)$, Germany $(n=6)$, India $(n=3)$, Thailand $(n=3)$, Egypt $(n=2)$, Argentina $(n=2)$, Turkey $(n=1)$, Italy $(n=1)$, Mexico $(n=1)$, Belgium $(n=1)$, Denmark $(n=1)$, Sweden $(n=1)$, Croatia $(n=1)$, UAE $(n=1)$ and Ireland $(n=1)$. Patients were investigated at their home institutions and the referring physicians completed a proforma detailing the clinical and biochemical data at the time of sending the DNA sample. The referring clinicians excluded causes of secondary GHI, including undernutrition. Birth weight, height and BMI were expressed as SDS according to the appropriate national standards. Biochemical investigations included: basal and/ or peak GH and basal IGF-1 levels. IGF-1 was expressed as SDS based on the age- and sex-appropriate range provided by the institution. Where serum IGF-1 was undetectable (less than the lower limit of the assay) $(n=17)$, we calculated the lowest possible detectable SDS and assigned that for the statistical analysis. In these patients, the IGF-1 SDS ranged between -2.5 and -5.3 , but this is likely to underestimate the degree of IGF-1 insensitivity.

Twenty-five of 132 patients did not have the clinical and biochemical characteristics of GH or IGF-1 insensitivity and were excluded (Fig. 1A). Diagnoses in the excluded group included GHD $(n=4)$, short stature associated with chromosome 10 duplication $(n=1)$ and achondroplasia (FGFR3 mutation) $(n=1)$. One hundred and seven cases (97 families, 97 probands) were investigated, including 
A

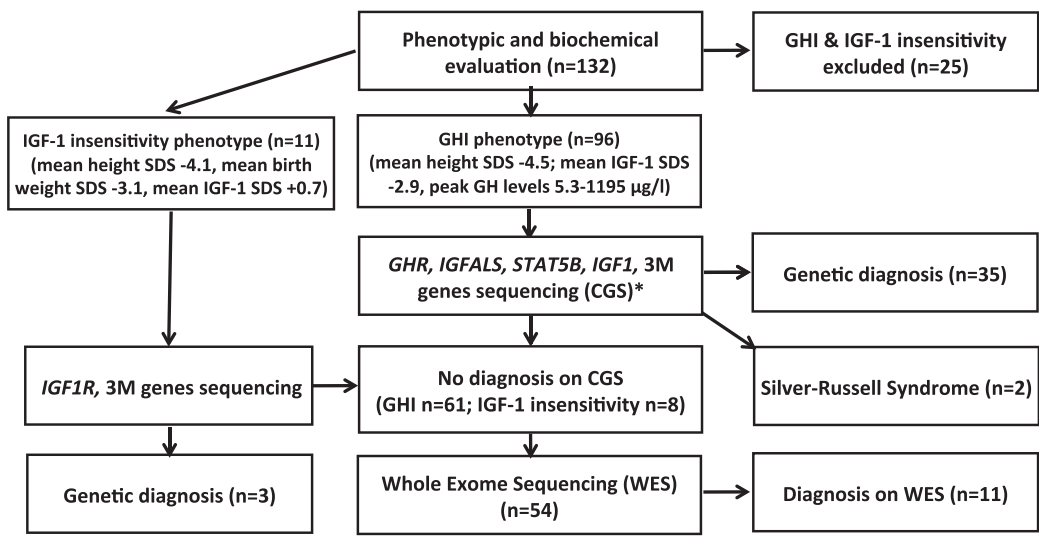

B

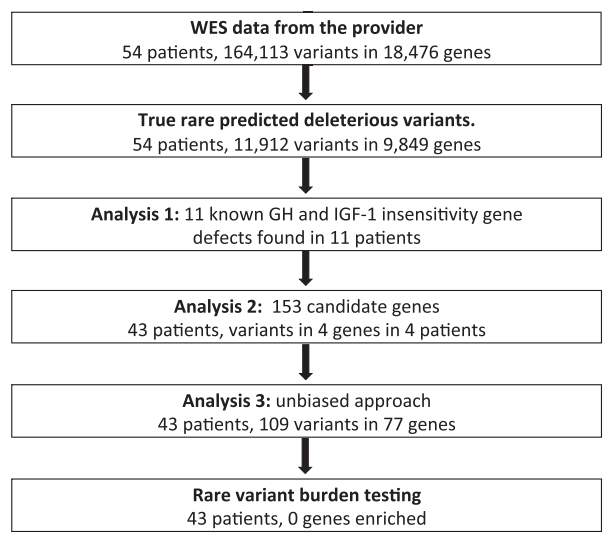

\section{Figure 1}

Summary of candidate gene (CGS) and whole-exome sequencing (WES) in the GH and IGF-1 insensitivity patients. (A) $3 M$ syndrome genes, CUL7, CCDC8 and OBSL1; BW, birth weight. *The candidate genes sequenced depended on the clinical and biochemical features. The majority of patients were screened for mutations in the growth hormone receptor gene (GHR) +/- IGFALS. Other genes were selected depending on the phenotype e.g. STAT5B if there was evidence of immune deficiency/ eczema/atopy and IGF1 and 3M genes if birth weight SDS was $\leq 2.0$ SDS. (B) Whole-exome sequencing (WES) data analyses: number of patients assessed and variants identified.

49 patients (42 probands) with consanguineous parents. Ninety-six cases (58M, median age: 5.8 years, range: 0.1-17.0) had features of GHI: mean height: -4.5 SDS (range: -9.1 to -2.0 ), mean IGF1: -2.9 SDS (range: -8.2 to -2.0 ) and peak GH levels: 7-1195 $\mu \mathrm{g} / \mathrm{L}$. A further 11 children (2M, median age: 5.8 years, range: $0.1-14.4)$ had characteristics of IGF-1 insensitivity: mean height SDS: -4.1 (range: -6.8 to -2.4 ), mean birth weight SDS: -3.1 (range: -5.8 to -2.0 ) and mean IGF-1 SDS: 0.7 (range: -1.1 to 4.4$)$.

\section{Candidate gene sequencing (CGS)}

Genomic DNA was isolated from peripheral blood leukocytes (Qiagen DNeasy Kit) and genetic analysis was undertaken on all patients as previously described (10). The candidate genes sequenced depended on the clinical and biochemical features. Most patients were screened for mutations in the growth hormone receptor gene (GHR); other genes were selected depending on the phenotype (Fig. 1A). Sanger sequencing was performed by the Barts and the London Genome Centre (http://www.smd.qmul. ac.uk/gc/) or GATC Biotech (https://www.gatc-biotech. com). Two patients underwent molecular investigations for Silver-Russell syndrome (SRS) following referral to clinical geneticists. Three patients with GHI had STAT5B sequencing.

\section{Whole-exome sequencing (WES)}

WES was completed in 54 patients (53 probands and 11 unaffected relatives) who had no genetic cause for their short stature identified by CGS (Fig. 1A). The remaining 15 patients did not consent for WES.

Twenty-three patients and 3 relatives were processed using the Agilent SureSelect all exon V4 capture and paired-end $(2 \times 100)$ sequencing on an Illumina HiSeq 2000 at Otogenetics (Norcross, GA, USA). 31 patients and 8 relatives were sequenced using SureSelect Human All Exon v5 $(51 \mathrm{Mb})$ capture and paired-end $(2 \times 100)$ sequencing on an Illumina 2500 Standard run (minimum coverage 50x) at Oxford Gene Technology (OGT, Oxford, UK). $>90 \%$ of target bases were covered $10 \times$. For comparison, WES data from 43 in-house controls generated on the same platforms were analysed by the same pipeline described below.

\section{Variant analysis}

The raw data from Otogenetics or OGT were analysed using DNA Nexus (DNAnexus Inc., Mountain View, CA, USA) by aligning to the H. sapiens GRCh37-b37 (1000 genomes Phase 1) reference genome with BWA-MEM FastQ Readmapper VCF files, generated by Vendor Human Exome GATK-Lite Variant Caller (Unified Genotyper). The 
resulting VCF files were uploaded to Ingenuity Variant Analysis (Qiagen; http://www.ingenuity.com/). The following filter settings were applied: call quality was set to $\geq 20$ and read depth $\geq 10$ and only data outside $0.1 \%$ of most exonically variable 100 base windows in healthy public genomes and outside $0.1 \%$ most exonically variable genes in healthy public genomes (1000 genomes, ExAC (http://exac.broadinstitute.org)) were included. Common variants were filtered out by excluding those with an allele frequency of $\geq 0.1 \%$ in the 1000 genomes, ExAC and the NHLBI exomes. Missense variations that were classified as loss of function by ingenuity were included i.e. the amino acid change was predicted to affect function and those that were predicted benign were excluded. Variants that passed these filters and were predicted damaging by either SIFT (http://sift.jcvi.org) or PolyPhen (http://genetics. bwh.harvard.edu/pph2/) were explored further (Fig. 1B):

\section{Analysis 1}

Variants were sought in 22 genes known to cause features of GHI or IGF-1 insensitivity (GHR, IGFALS, STAT5B, IGF1, PAPPA2, IGF1R, OBSL1, CCDC8, CUL7, PTPN11, SOS1, SOS2, RAF1, BRAF, NRAS, KRAS, HRAS, CBL, RIT1, NF1, LZTR1 and SHOC2). Genetic variants were confirmed by Sanger sequencing (SS), primer sequences available on request. Forty-five family members underwent SS to assess the segregation of the variant within family structures. If no putative causal variants were found, we progressed to Analysis 2.

\section{Analysis 2}

Variants were sought in 153 biological candidate genes associated with syndromic growth disorders, skeletal dysplasias, growth plate biology, cell proliferation, DNA repair or growth retardation in mice (Supplementary Table 1, see section on supplementary data given at the end of this article). An autosomal recessive model was adopted i.e. homozygous, hemizygous (for X-linkage) or potentially compound heterozygous variants as there were no affected parents. Variants were only included if they were present in patients and absent in controls. Since the cohort is genetically and phenotypically heterogeneous, we hypothesised that the same causal variant was unlikely to be seen in multiple patients (barring related individuals). Therefore, any variants that were present in $\geq 3$ patients were discarded. If no putative causal variants were identified by these criteria we progressed to Analysis 3.

\section{Analysis 3}

Variants were sought in novel candidate genes by an unbiased approach, seeking homozygous or putative compound heterozygous variants. Novel candidate genes were included if predicted deleterious variants were identified in $\geq 2$ patients and were absent in controls. Although this strategy may miss private mutations, it provides corroborative evidence that the gene is implicated in the phenotype. As mentioned previously, identical variants that were present in $\geq 3$ patients were discarded. Candidate genes satisfying these bioinformatic criteria were investigated in silico (see novel variants).

\section{Rare variant burden testing}

Rare variant burden testing was applied to the pre-filtered variants from Analysis 3 to identify genes enriched for rare variants in patients but not controls. The following script was employed using freeware $\mathrm{R}$ (https://cran.rproject.org/):

Table $<$-read.Table $($ file $="$ genes.txt", head=FALSE $)$ \#imports file genes

apply(Table,1, function(Table) fisher. test(matrix(Table,nr=2))\$P.value)

\section{Novel variants}

Novel variants were investigated in silico by SIFT (score ranges from 0 (predicted deleterious) to 1 (predicted benign)), PolyPhen-2 (score ranges from 0 (predicted benign) to 1 (predicted deleterious)), variant effect predictor (VEP), Mutation Taster (www.mutationtaster. org) and Human Splicing Finder (HSF, version 3.0; http:// www.umd.be/HSF3/) to predict the functional outcome. VEP (http://grch37.ensembl.org/Homo_sapiens/Tools/ VEP) defines the likely deleterious effect of the variant as low, moderate or high. Mutation Taster predicts whether the variant is predicted disease causing or benign. HSF predicts whether a variant makes exon skipping more likely than the reference allele. PubMed (https://www. ncbi.nlm.nih.gov/pubmed/), OMIM (http://www.omim. org/) and String (http://string-db.org) determined Gene function and for pathway analysis.

\section{Statistical analysis}

The differences in height SDS, IGF-1 SDS and peak GH between those with and without an identified genetic defect were analysed using an unpaired $t$-test. Univariate 
logistic regression analysis identified predictor variables (SPSS, version 22; IBM).

\section{Ethics}

Informed written consent for genetic research was obtained from patients and/or their parents.

\section{Results}

\section{Diagnosis by candidate gene sequencing (CGS)}

CGS identified likely causative variants in 35 GHI patients (28 probands) and 3 IGF-1 insensitivity patients, all probands (total $38 / 107 ; 36 \%$ or $31 / 97,32 \%$ probands) (Table 1). These included variants in $G H R$ ( $n=27$ patients), IGFALS ( $n=3$ patients), OBSL1 ( $n=6$ patients), CUL7 ( $n=1$ patients) and $\operatorname{IGF1R}$ ( $n=1$ patient) (Figs $1 \mathrm{~A}$ and 2$) .30$ of 38 (79\%) children diagnosed by CGS had consanguineous parents. STAT5B sequencing was normal in the 3 patients tested.

\section{GHR}

Fifteen GHR variants (5 novel and 10 previously described) were identified in 27 patients (patients 1-27, Table 1) with mean serum IGF-1: -3.5 SDS (range: -8.5 to -2.3 ), mean basal and peak GH concentrations: $40.7 \mu \mathrm{g} / \mathrm{L}$ (range: $1.8-$ $398, n=21$ ) and $123.2 \mu \mathrm{g} / \mathrm{L}$ (range: $15.7-1195, n=20)$. All had homozygous variants in GHR with the exception of 2 patients (21 and 26) who had compound heterozygous variants, inheriting one defective allele from each parent. All children except patient 1 had a 'classical' Laron syndrome phenotype. The most commonly identified GHR defect was the homozygous $6 \psi$ mutation (c.618+792A $>\mathrm{G}$, p.Met206_Met207ins36) in patients 1-8 of UK Pakistani or Indian origin. These included two unrelated pairs of siblings (patients $2 \& 3,5 \& 6$ ) and four other non-familial cases (patients 1, 4, 7, 8). Patient 1 had a GHR intronic pseudoexon $(6 \psi)$ mutation with characteristic features of GHI (height SDS: -4.0, IGF1 SDS: -2.6 , peak GH levels: $119 \mu \mathrm{g} / \mathrm{L})$ but no dysmorphic features. Four of the novel GHR variants were homozygous; c.198C>A (p.Cys66*), c.700C $>\mathrm{T} \quad($ p.Gln234*), c.599A $>\mathrm{G} \quad$ (p.Asn200Ser) c.344A $>$ C (p.Asn115Thr) all predicted deleterious by at least one functional outcome prediction method. Patient 21 had 2 previously described variants in compound heterozygosity (c.266+83G $>\mathrm{T}$ (p.?) and (c.723C $>\mathrm{T}$, p.Gly241_Glu261del). The final novel variant, c.922G>A (p.Gly308Arg) predicted deleterious by SIFT, was found in compound heterozygosity with a known GHR variant in patient 26. Other known GHR mutations identified were: c.740T>C (p.Leu247Pro), c.594A>G (p.Glu198*), c.785-6 T>A (p.Asp264Glyfx*), c.247C>T (p.Gln83*), c.703C>T (p.Arg235*), c.439+1 G>A (p.Arg89Serfs*47), c.723C>T (p.Gly241_Glu261del) and c.168C>A (p.Cys56*).

\section{IGFALS}

Three GHI patients $(28,29,30)$ had homozygous IGFALS variants (mean serum IGF1 SDS $-2.7(-3.6$ to -1.9$)$ and mean peak GH concentration $20.5 \mu \mathrm{g} / \mathrm{L}(16.0-28.9 \mu \mathrm{g} / \mathrm{L})$. One variant c.1291delT, pTrp431Glyfs*11 has been previously described (10). Interestingly, the previously described p.Leu134Gln variant identified in 2 patients (28 \& 29) was associated with SGA but no dysmorphic features (10).

\section{M syndrome genes}

We identified 2 previously described homozygous OBSL1 mutations c.1463C>T (p.Arg489*) (patients 31 and 32) and c.1359insA, (p.Glu454Argfs*) (patients 33-36) and 1 homozygous CUL7 c.2710C>T (p.Arg904*) mutation (patient 37) $(23,24)$. All patients had consanguineous parents. All patients had severe short stature (mean height SDS -5.8) with normal GH (mean peak GH: 21.8). Most had severe IGF-1 deficiency but 2 (patients 35 and 37) had IGF-1 levels of -0.2 and -0.25 , respectively. Additional but variable clinical features of the $3 \mathrm{M}$ syndrome were present in all 7 patients (Table 1).

\section{IGF1R}

A heterozygous missense variant was identified in one patient with an IGF-1 insensitivity phenotype (birth weight -2.7 SDS, height SDS -3.1, IGF-1 SDS 2.0, basal $\mathrm{GH} 17.5 \mu \mathrm{g} / \mathrm{L}$ ) (patient 38). This heterozygous variant, c.112G>A, (p.Asp38Asn) has previously been described (Table 1) (10).

\section{Silver-Russell syndrome}

Hypomethylation in the imprinting control region 11 p15 and mUPD7 was demonstrated in patients 39 and 40 , respectively. Both had features of GHI as previously described (frontal bossing, mid-facial hypoplasia, height SDS: -3.7 and -4.3 , and IGF-1 SDS: -2.8 and -3.4 ) (Fig. 1A and Supplementary Table 2) (10). 
Table 1 Clinical, biochemical and genetic features of patients diagnosed by candidate gene sequencing (CGS) (total 37 patients, 39 variants).

\begin{tabular}{|c|c|c|c|c|c|c|c|c|c|c|c|}
\hline $\begin{array}{l}\text { Patient } \\
\text { number }\end{array}$ & Age (year) & Sex & $\begin{array}{l}\text { Consanguinity/ } \\
\text { ethnicity }\end{array}$ & $\begin{array}{c}\text { Birth } \\
\text { weight } \\
\text { SDS }\end{array}$ & $\begin{array}{l}\text { Height } \\
\text { SDS }\end{array}$ & BMI SDS & $\begin{array}{c}\text { Target } \\
\text { Height SDS }\end{array}$ & $\begin{array}{c}\text { GH basal } \\
(\mu \mathrm{g} / \mathrm{L})\end{array}$ & GH $\max (\mu \mathrm{g} / \mathrm{L})$ & $\begin{array}{c}\text { IGF-1 } \\
\text { (ng/mL) }\end{array}$ & IGF-1 SDS \\
\hline 1 & 8.0 & M & +/Pakistani & -0.5 & -4.0 & 0.7 & -0.9 & 13.2 & 119.0 & 18.2 & -2.6 \\
\hline $2^{5}$ & 4.2 & $\mathrm{~F}$ & +/Pakistani & 0.1 & -4.2 & -1.0 & 0.7 & 16.3 & 33.3 & $<22.4$ & $-2.5^{\mathrm{a}}$ \\
\hline $3^{\mathrm{s}}$ & 7.5 & M & +/Pakistani & -2.9 & -4.5 & -1.2 & -1.3 & 4.0 & $>33$ & 1.4 & -2.8 \\
\hline 4 & 7.7 & M & +/Indian & -1.7 & -3.1 & -2.4 & -1.9 & 3.2 & 30.3 & 11.2 & -2.6 \\
\hline $5^{B}$ & 14.7 & $M$ & +/Pakistani & 0.7 & -3.0 & -0.7 & -1.0 & 11.3 & 39.6 & 9.1 & -3.1 \\
\hline $6^{\mathrm{B}}$ & 2.3 & $M$ & +/Pakistani & NK & -4.7 & -0.5 & -1.0 & 50.8 & 46.0 & $<22.4$ & $-3.1^{\mathrm{a}}$ \\
\hline 7 & 2.4 & $\mathrm{~F}$ & +/Pakistani & -1.8 & -5.0 & -0.4 & $N / D$ & 3.4 & 26.7 & 134.3 & -2.3 \\
\hline 8 & 6.8 & $\mathrm{~F}$ & +/Pakistani & -0.3 & -4.1 & -0.2 & -0.9 & 56.1 & 30.3 & 30.3 & -4.0 \\
\hline $9^{b}$ & 2.4 & M & -/Argentinian & 0.2 & -7.7 & -0.8 & -1.9 & NK & 57.0 & UD & $-2.5^{a}$ \\
\hline $10^{\mathrm{b}}$ & 14.3 & $M$ & -/Argentinian & 0.5 & -8.4 & 0.0 & -1.9 & NK & 88.0 & 9.0 & NK \\
\hline 11 & 8.4 & M & +/Turkish & -6.0 & -8.7 & 1.8 & -1.9 & NK & 79.0 & 5.1 & NK \\
\hline 12 & 5.8 & $M$ & $+/$ NK & -0.7 & -7.7 & -0.7 & -1.4 & NK & 3344344A32.5 & UD & $-2.5^{\mathrm{a}}$ \\
\hline 13 & NK & M & NK/Mexican & NK & NK & NK & NK & NK & NK & NK & NK \\
\hline 14 & 4.2 & M & +/Bangladeshi & 1.6 & -6.9 & -5.6 & -1.8 & 398.0 & 1195.0 & $<25$ & $-2.5^{\mathrm{a}}$ \\
\hline 15 & 1.8 & $\mathrm{~F}$ & +/Bangladeshi & -0.2 & -5.7 & -2.2 & -2.3 & 36.0 & ND & $<25$ & $-2.5^{\mathrm{a}}$ \\
\hline 16 & 1.5 & $\mathrm{~F}$ & +/Bangladeshi & -1.2 & -6.1 & 0.7 & -2.0 & 60.0 & ND & $<25$ & $-2.5^{a}$ \\
\hline 17 & 1.1 & M & +/Kuwaiti & 2.6 & -5.0 & 0.8 & -0.3 & $>47$ & $>47$ & $<30$ & $-2.5^{a}$ \\
\hline 18 & 5.7 & M & +/Kuwaiti & -0.6 & -5.3 & 1.7 & -1.3 & 14.4 & 35.0 & 32.9 & $-2.5^{\mathrm{a}}$ \\
\hline $19^{c}$ & 9.4 & $M$ & +/Egyptian & 2.1 & -6.4 & -5.5 & -1.5 & 16.0 & 33.3 & 9.5 & -8.5 \\
\hline $20^{c}$ & 6.2 & $M$ & +/Egyptian & 2.1 & -5.5 & -2.8 & -1.5 & 1.8 & 15.7 & 8.1 & -8.2 \\
\hline 21 & 10.5 & M & -/Caucasian & 1.5 & -2.9 & 1.8 & 1.0 & 42.2 & ND & 42.0 & -3.0 \\
\hline $22^{d}$ & 15.3 & $\mathrm{~F}$ & $-/$ Thai & -2.3 & -9.1 & -1.3 & -2.15 & ND & 41 & 67.5 & -2.5 \\
\hline $23^{d}$ & 5.8 & $\mathrm{~F}$ & $-/$ Thai & -1.8 & -6.9 & -1.0 & -2.15 & 15.9 & 108.44 & $<1$ & $-2.8^{a}$ \\
\hline $24^{e}$ & 4.0 & M & +/Indian & -1.3 & -7.9 & -1.3 & -2.62 & 12.4 & ND & $<25$ & $-3.1^{\mathrm{a}}$ \\
\hline $25^{e}$ & 1.3 & $M$ & +/Indian & -2.6 & -8.9 & -2.0 & -2.62 & 24.5 & ND & ND & ND \\
\hline
\end{tabular}




\begin{tabular}{|c|c|c|c|}
\hline \multirow[b]{2}{*}{ Clinical features } & \multicolumn{3}{|c|}{ Genetic variants } \\
\hline & GHR gene & IGFALS gene & $\begin{array}{l}\text { 3M syndrome } \\
\text { genes }\end{array}$ \\
\hline No & $\begin{array}{l}\text { Hom c. } 618+792 A>G \\
\text { p.Met206_ } \\
\text { Met207ins36 }\end{array}$ & & \\
\hline Classical & $\begin{array}{l}\text { Hom c. } 618+792 A>G, \\
\text { p.Met206_- } \\
\text { Met207ins36 }\end{array}$ & & \\
\hline Classical & $\begin{array}{l}\text { Hom c.618+792A>G, } \\
\text { p.Met206_ } \\
\text { Met207ins36 }\end{array}$ & & \\
\hline Classical & $\begin{array}{l}\text { Hom c. } 618+792 A>G, \\
\text { p.Met206_ } \\
\text { Met207ins36 }\end{array}$ & & \\
\hline Classical & $\begin{array}{l}\text { Hom c. } 618+792 A>G \\
\text { p.Met206_ } \\
\text { Met207ins36 }\end{array}$ & & \\
\hline Classical & $\begin{array}{l}\text { Hom c. } 618+792 A>G, \\
\text { p.Met206_ } \\
\text { Met207ins36 }\end{array}$ & & \\
\hline Classical & $\begin{array}{l}\text { Hom c. } 618+792 A>G \\
\text { p.Met206_ } \\
\text { Met207ins36 }\end{array}$ & & \\
\hline Classical & $\begin{array}{l}\text { Hom c.618+792A>G, } \\
\text { p.Met206 } \\
\text { Met207ins36 }\end{array}$ & & \\
\hline Classical & $\begin{array}{l}\text { Hom c. } 198 \mathrm{C}>\mathrm{A} \\
\text { p.Cys66* }\end{array}$ & & \\
\hline Classical & Homc. $198 \mathrm{C}>$ A, p.Cys66* & & \\
\hline Classical & $\begin{array}{l}\text { Hom } c .700 C>T, \\
\text { p.Gln234* }\end{array}$ & & \\
\hline Classical & $\begin{array}{l}\text { Hom c.740T>C, } \\
\text { p.Leu247Pro (p.L229P) }\end{array}$ & & \\
\hline Classical & $\begin{array}{l}\text { Hom c.594A>G, } \\
\text { p.Glu198* (E180X) }\end{array}$ & & \\
\hline Classical & $\begin{array}{l}\text { Hom c.785-6 T>A, } \\
\text { p.Asp264Glyfx*5 } \\
\text { (p.D244GfsX5) }\end{array}$ & & \\
\hline Classical & $\begin{array}{l}\text { Hom c.247C }>T, \text { p.Gln83* } \\
(\mathrm{Q} 65 \mathrm{X})\end{array}$ & & \\
\hline Classical & $\begin{array}{l}\text { Hom c. } 703 \mathrm{C}>\mathrm{T} \\
\text { p.Arg235* } \\
\text { R217X) }\end{array}$ & & \\
\hline Classical & $\begin{array}{l}\text { Hom c.703C >T, } \\
\text { p.Arg235* (R217X) }\end{array}$ & & \\
\hline Classical & $\begin{array}{l}\text { Hom c. } 703 \mathrm{C}>\mathrm{T} \\
\text { p.Arg235* } \\
\text { (R217X) }\end{array}$ & & \\
\hline Classical & $\begin{array}{l}\text { Hom c.439+1 G>A, } \\
\text { p.Arg89Serfs*47 }\end{array}$ & & \\
\hline Classical & $\begin{array}{l}\text { Hom c.439+1 G>A, } \\
\text { p.Arg89Serfs*47 }\end{array}$ & & \\
\hline Classical & c.266+83G>T, p.? & & \\
\hline & $\begin{array}{l}\text { c.723C> T, p.Gly241_ } \\
\text { Glu261del (p.G223_ } \\
\text { E243del) }\end{array}$ & & \\
\hline Classical & $\begin{array}{l}\text { Hom. c.723C>T, } \\
\text { p.Gly241_Glu261del } \\
\text { (p.G223_E243del) }\end{array}$ & & \\
\hline Classical & $\begin{array}{l}\text { Hom. c.723C>T, } \\
\text { p.Gly241_Glu261del } \\
\text { (p.G223_E243del) }\end{array}$ & & \\
\hline Classical & $\begin{array}{l}\text { Hom c.599A>G, } \\
\text { p.Asn200Ser }\end{array}$ & & \\
\hline Classical & $\begin{array}{l}\text { Hom c.599A }>\text { G, } \\
\text { p.Asn200Ser }\end{array}$ & & \\
\hline
\end{tabular}

\begin{tabular}{|c|c|c|c|}
\hline $\begin{array}{l}\text { MAF } \\
\text { ExAC }\end{array}$ & $\begin{array}{c}\text { Predicted } \\
\text { outcome (novel } \\
\text { variants) }\end{array}$ & Reference & $\begin{array}{l}\text { No. genes } \\
\text { analysed } \\
\text { by CGS }\end{array}$ \\
\hline 0 & - & (26) & $1(G H R)$ \\
\hline 0 & - & (26) & $1(G H R)$ \\
\hline 0 & - & (26) & $1(G H R)$ \\
\hline 0 & - & (26) & $1(G H R)$ \\
\hline 0 & - & (26) & $1(G H R)$ \\
\hline 0 & - & (26) & $1(G H R)$ \\
\hline 0 & - & (26) & $1(G H R)$ \\
\hline 0 & - & (26) & $1(G H R)$ \\
\hline 0 & High impact (VEP) & Unpublished & $1(G H R)$ \\
\hline 0 & High impact (VEP) & Unpublished & $1(G H R)$ \\
\hline 0 & High impact (VEP) & Unpublished & $1(G H R)$ \\
\hline 0 & $\begin{array}{l}\text { Deleterious (SIFT } \\
\text { score 0) }\end{array}$ & (35) & $1(G H R)$ \\
\hline 0 & - & (36) & $1(G H R)$ \\
\hline 0 & - & (37) & $1(G H R)$ \\
\hline 0 & - & (38) & $1(G H R)$ \\
\hline $8.2 \times E^{-6 y}$ & - & (39) & $1(G H R)$ \\
\hline $8.2 \times E^{-6} \mathrm{y}$ & - & (39) & $1(G H R)$ \\
\hline $8.2 \times \mathrm{E}^{-6 \mathrm{y}}$ & - & (39) & $1(G H R)$ \\
\hline 0 & - & (10) & $1(G H R)$ \\
\hline 0 & - & (10) & $1(G H R)$ \\
\hline 0 & - & (40) & $1(G H R)$ \\
\hline 0 & - & (41) & \\
\hline 0 & - & (41) & $1(G H R)$ \\
\hline 0 & - & (41) & $1(G H R)$ \\
\hline 0 & Deleterious (SIFT) & Unpublished & $1(G H R)$ \\
\hline 0 & Deleterious (SIFT) & Unpublished & $1(G H R)$ \\
\hline
\end{tabular}

(Continued) 
Table 1 Continued.

\begin{tabular}{|c|c|c|c|c|c|c|c|c|c|c|c|}
\hline $\begin{array}{l}\text { Patient } \\
\text { number }\end{array}$ & Age (year) & Sex & $\begin{array}{l}\text { Consanguinity/ } \\
\text { ethnicity }\end{array}$ & $\begin{array}{c}\text { Birth } \\
\text { weight } \\
\text { SDS }\end{array}$ & $\begin{array}{l}\text { Height } \\
\text { SDS }\end{array}$ & BMI SDS & $\begin{array}{c}\text { Target } \\
\text { Height SDS }\end{array}$ & $\begin{array}{c}\text { GH basal } \\
(\mu \mathrm{g} / \mathrm{L})\end{array}$ & GH $\max (\mu \mathrm{g} / \mathrm{L})$ & $\begin{array}{c}\text { IGF-1 } \\
(\mathrm{ng} / \mathrm{mL})\end{array}$ & IGF-1 SDS \\
\hline 26 & 1.6 & $\mathrm{~F}$ & -/Caucasian & -1.4 & -4.3 & -1.4 & NK & 25.5 & ND & $<25$ & $-2.5^{\mathrm{a}}$ \\
\hline 27 & 3.1 & $\mathrm{~F}$ & +/Arabic-Syrian & -1.1 & -4.5 & -1.2 & -1.02 & 3.3 & 75.0 & 8.0 & -7.3 \\
\hline $28^{\mathrm{s}}$ & 8.0 & $\mathrm{~F}$ & +/Indian & -3.0 & -3.9 & -2.5 & -2.32 & 2.2 & 28.9 & $<16.0$ & $-2.5^{\mathrm{a}}$ \\
\hline $29^{\mathrm{s}}$ & 6.1 & M & +/Indian & -2.1 & -2.0 & NK & -4.1 & 1.1 & 16.7 & 25.9 & -1.9 \\
\hline 30 & 13.5 & $\mathrm{~F}$ & +/Moroccan & -1.3 & -3.3 & -1.75 & -1.63 & 9.7 & 16.01 & 66.0 & -3.6 \\
\hline 31 & 4.6 & $M$ & +/Bedouin & -3.2 & -7.4 & -0.7 & -0.5 & 6.0 & 32 & 6.4 & -2.5 \\
\hline 32 & 1.0 & $M$ & +/Kuwaiti & -1.6 & -6.4 & -2.3 & -1.3 & 2.1 & 18.2 & 30.5 & -2.5 \\
\hline 33 & 3.0 & $\mathrm{~F}$ & +/Kuwaiti & -5.2 & -5.7 & -4.7 & NK & 9.1 & 15 & $<3.0$ & $-2.7^{a}$ \\
\hline $34^{9}$ & 1.1 & $\mathrm{~F}$ & +/Kuwaiti & -3.8 & -4.9 & -0.4 & -0.34 & 4.2 & 37.2 & ND & ND \\
\hline $35^{9}$ & 0.1 & $\mathrm{~F}$ & +/Kuwaiti & -2.6 & -5.1 & 0.7 & -0.34 & 5.4 & 10.8 & 105.0 & -0.2 \\
\hline 36 & 0.06 & $\mathrm{~F}$ & +/Kuwaiti & -1.5 & -4.5 & 0.5 & -1.2 & 41.0 & 33 & $<27$ & -2.6 \\
\hline 37 & 10.8 & $\mathrm{~F}$ & +/Bangladeshi & -2.9 & -6.8 & 1.0 & -2.39 & NK & 6.5 & 159.0 & -0.3 \\
\hline 38 & 6.6 & $\mathrm{~F}$ & -/Caucasian & -2.7 & -3.1 & -1.3 & -0.46 & 17.5 & 9.6 & 367.0 & 2.0 \\
\hline
\end{tabular}

\section{Diagnosis by whole-exome sequencing (WES)}

164,113 variants in 18,476 genes were called in 54 patient exomes (53 probands). Following the application of the filters described above for true rare predicted deleterious changes, this reduced to 11,912 variants in 9849 genes (Fig. 1B).

\section{Analysis 1}

Figure 2 and Table 2: 11/54 patients (20\%) (10 probands, 19\%) were found to have variants in genes known to cause GHI (homozygous GHR $(n=5)$, compound heterozygous IGFALS $(n=1)$, homozygous CCDC8 $(n=1)$, homozygous CUL7 $(n=1)$, heterozygous PTPN11 $(n=2)$ and heterozygous SOS1 $(n=1))$. 


\begin{tabular}{|c|c|c|c|c|c|c|c|}
\hline & & Genetic variant & & & & & \\
\hline Clinical features & GHR gene & IGFALS gene & $\begin{array}{l}\text { 3M syndrome } \\
\text { genes }\end{array}$ & $\begin{array}{l}\text { MAF } \\
\text { ExAC }\end{array}$ & $\begin{array}{c}\text { Predicted } \\
\text { outcome (novel } \\
\text { variants) }\end{array}$ & Reference & $\begin{array}{c}\text { No. genes } \\
\text { analysed } \\
\text { by CGS }\end{array}$ \\
\hline \multirow[t]{2}{*}{ Classical } & $\begin{array}{l}\text { c. } 168 \mathrm{C}>\mathrm{A}, \mathrm{p} . \text { Cys56* (p. } \\
\text { C38X) }\end{array}$ & & & $8.2 \times E^{-6 x}$ & - & (42) & $1(G H R)$ \\
\hline & c.922G $>$ A, p.Gly308Arg & & & 0 & $\begin{array}{l}\text { Deleterious (SIFT } \\
\text { score 0) }\end{array}$ & Unpublished & \\
\hline Classical & $\begin{array}{l}\text { Hom c.344A>C,p. } \\
\text { Asn115Thr }\end{array}$ & & & 0 & Deleterious (SIFT) & Unpublished & $1(G H R)$ \\
\hline No & & $\begin{array}{l}\text { Hom c.401T>A } \\
\text { p.Leu134Gln } \\
\text { (p.L134Q) }\end{array}$ & & & - & (43) & $\begin{array}{l}2 \text { (GHR, } \\
\quad \text { IGFALS) }\end{array}$ \\
\hline No & & $\begin{array}{l}\text { Hom c.401T>A } \\
\text { p.Leu134GIn } \\
\text { (p.L134Q) }\end{array}$ & & & - & (43) & 1 (IGFALS) \\
\hline No & & $\begin{array}{l}\text { Hom c.1291delT, } \\
\text { pTrp431Glyfs*11 } \\
\text { (p.W431Gfs11) }\end{array}$ & & 0 & - & (10) & $\begin{array}{l}2(G H R \\
\quad I G F A L S)\end{array}$ \\
\hline Classical & & & $\begin{array}{l}\text { OBSL1 Hom c. 1463C >T, } \\
\text { p.Arg489* (p.R489X) }\end{array}$ & 0 & - & (23) & $\begin{array}{r}2 \text { (IGF1R, } \\
\text { OBSL1) }\end{array}$ \\
\hline Classical & & & OBSL1 Hom c. $1463 \mathrm{C}>\mathrm{T}$ & 0 & - & (23) & $4(G H R$ \\
\hline $\begin{array}{l}\text { Hypermobility, } \\
\text { prominent heels }\end{array}$ & & & p.Arg489* (p.R489X) & & & & $\begin{array}{l}\text { IGFALS, } \\
\text { IGF1, } \\
\text { OBSL1) }\end{array}$ \\
\hline $\begin{array}{l}\text { Classical } \\
\text { Hypermobility, } \\
\text { prominent heels }\end{array}$ & & & $\begin{array}{l}\text { OBSL1 Hom c.1359insA, } \\
\text { p.Glu454Argfs } * 11 \\
\text { (p.E454RfsX11) }\end{array}$ & 0 & - & (23) & $\begin{array}{l}2 \text { (IGF1, } \\
\text { OBSL1) }\end{array}$ \\
\hline $\begin{array}{l}\text { Classical. } \\
\text { Hypermobility }\end{array}$ & & & $\begin{array}{l}\text { OBSL1 Hom c.1359insA, } \\
\text { p.Glu454Argfs } \star 11 \\
\text { (p.E454Rfs X11) }\end{array}$ & 0 & - & (23) & $\begin{array}{l}2 \text { (IGF1, } \\
\text { OBSL1) }\end{array}$ \\
\hline $\begin{array}{l}\text { Classical Prominent } \\
\text { heels }\end{array}$ & & & $\begin{array}{l}\text { OBSL1 Hom c.1359insA, } \\
\text { p.Glu454Argfs } * 11 \\
\text { (p.E454RfsX11) }\end{array}$ & 0 & - & (23) & $\begin{array}{r}2 \text { (IGF1R, } \\
\text { OBSL1) }\end{array}$ \\
\hline $\begin{array}{l}\text { Classical } \\
\text { hypermobility, } \\
\text { prominent heels } \\
\text { short fingers, } \\
\text { trident hands, } \\
\text { short rib cage, } \\
\text { bilateral DDH }\end{array}$ & $\begin{array}{l}\text { OBSL1 Hom c.1359insA, } \\
\text { p.E454Rfs*11 } \\
\text { (p.E454RfsX11) }\end{array}$ & & & 0 & - & (23) & $\begin{array}{l}4 \text { (GHR, } \\
\text { IGFALS, } \\
\text { IGF1, } \\
\text { OBSL1) }\end{array}$ \\
\hline $\begin{array}{l}\text { Disproportion } \\
\text { large head, short } \\
\text { limbs, lumbar } \\
\text { lordosis }\end{array}$ & $\begin{array}{l}\text { CUL7 Hom c. } 2710 \mathrm{C}>\mathrm{T} \text {, } \\
\text { p.Arg904* }\end{array}$ & & & 0 & - & (44) & $\begin{array}{c}3 \text { (IGF1R, } \\
\text { OBSL1, } \\
\text { CUL7) }\end{array}$ \\
\hline & IGF1R gene & & & & & & \\
\hline $\begin{array}{l}\text { Triangular face, } \\
\text { long fingers }\end{array}$ & $\begin{array}{l}\text { Het c.112G>A, } \\
\text { p.Asp38Asn (p.D38N) }\end{array}$ & & & 0 & - & (10) & 1 (IGF1R) \\
\hline
\end{tabular}

Novel genetic variants are in bold font. Height SDS is at presentation. ND, not done; NK, not known; UD, undetectable; +, parents consanguineous; -, parents not consanguineous; classical, classical GHI phenotype (frontal bossing, mid-facial hypoplasia); No, no dysmorphic features; DDH, developmental dysplasia of the hip; a, IGF-1 level less than the lower limit of the assay (SDS -2.5); Hom, homozygous; Het, heterozygous; VEP, variant effect predictor; S/s, siblings; $\mathrm{B} / \mathrm{b} / \mathrm{cle}$, brothers; $\mathrm{d} / \mathrm{g}$, sisters. 3M syndrome genes, CUL7, CCDC8 and OBSL1. c. coding DNA sequence where nucleotide 1 is the A of the ATG-translation initiation codon, for GHR the transcript includes exon 3 NCBI Reference Sequences NM_000163; for IGFALS NM_004970; for CUL7 NM_014780; for OBSL1 NM_015311; for IGF1R NM_000875; **, p.Met188_Met189ins36 mutation aka pseudoexon activation (6Y); ins, insertion; fs, frameshift; *, termination site; as, acceptor site; ds, donor site; X, stop codon; del, deletion; ${ }^{\beta}$ predicted result if exon is skipped. c, coding DNA sequence where nucleotide 1 is the A of the ATG-translation initiation codon of OBSL1 gene, NCBI reference NM_015311.2; MAF, minor allele frequency - variants are defined as rare if the MAF is $<0.001(0.1 \%)$ as recorded on the ExAC (Exome Aggregation Consortium) database; ${ }^{\mathrm{y}}$, no homozygotes in ExAC database. Variation predicted by HSF, HSF predicts exon skipping to be more likely than in reference allele; SIFT score 0 is deleterious, 1 is benign. References refer to the genetic variants. No. genes sequenced by CGS, number of genes (and which genes) sequenced by candidate gene sequencing (CGS) before a diagnosis was made. Variant nomenclature is according to the HGVS (http://varnomen.hgvs.org/) guidelines. Italicised patient numbers indicate those patients previously reported in Storr et al. 2015 (10). 


\section{CGS}

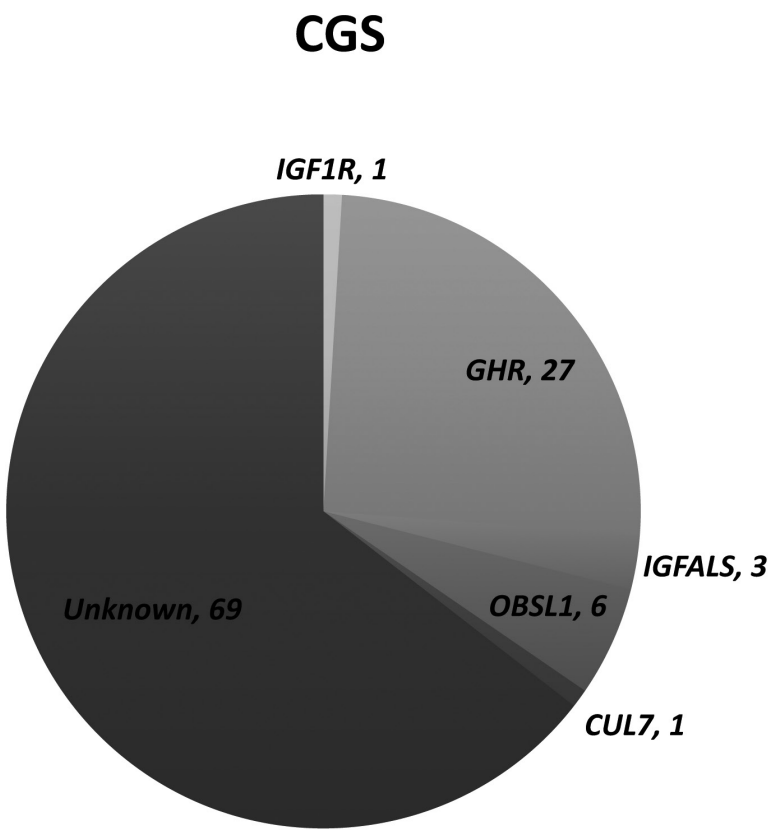

\section{$(38 / 107) 36 \%$}

\section{Figure 2}

Genetic diagnoses in the GH and IGF-1 insensitivity patients.

\section{GHR}

Patients 47-51 (Table 2) with GHR variants had classical Laron phenotypes (mean height SDS: -5.1 , mean IGF-1 SDS: -4.7 and mean peak GH: $46.8 \mu \mathrm{g} / \mathrm{L})$. Patients 47-50 had a novel homozygous GHR variant c.70+4A>C (p.?) (exon skipping predicted by HSF) and were from consanguineous families of Kuwaiti origin, therefore a founder effect is likely. Patient 51 had a previously described GHR c.703C > T, p.Arg235* (R217X) mutation, and had a clinical picture of classical Laron syndrome with height SDS -5.9, IGF-1 of -5.3 , and peak $\mathrm{GH}>35$.

\section{IGFALS}

Patient 46 (Table 2) with novel compound heterozygous IGFALS variants c. $1576 \mathrm{G}>\mathrm{A}$ (p.Asp526Asn) and c.632G >A, (p.Trp211*), both predicted deleterious (SIFT score 0), had a typical phenotype (height SDS: -5.0 , IGF1 SDS: -2.5 and peak GH: $13 \mu \mathrm{g} / \mathrm{L})$.

\section{Noonan syndrome (NS) genes}

Patients 41 and 42 had previously described heterozygous PTPN11 c.417G >C (p.Glu139Asp) and c.853T >C

\section{All modalities}

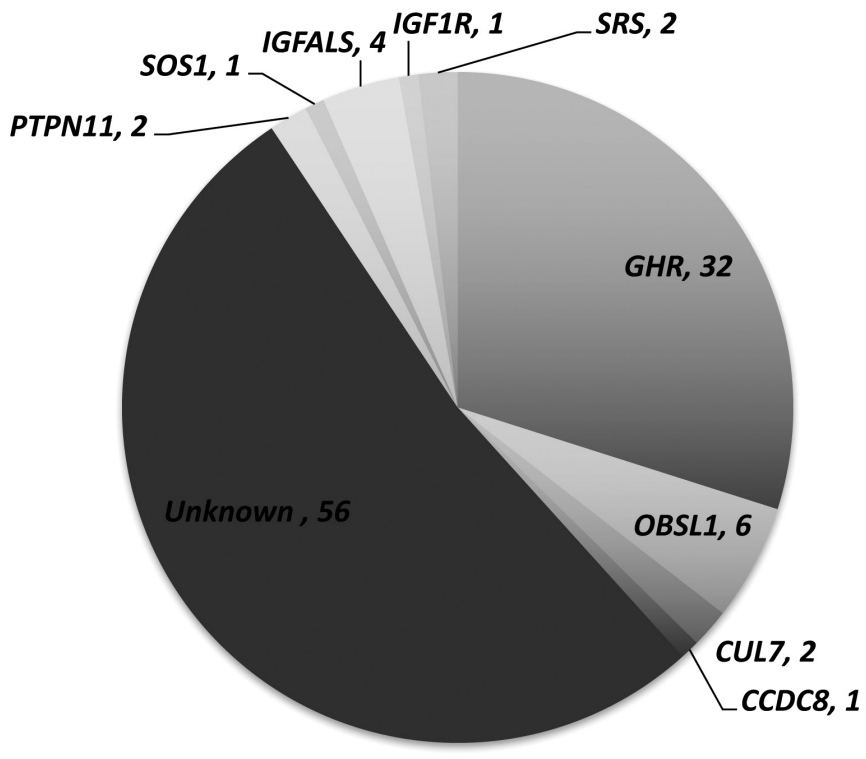

$(51 / 107) 48 \%$

(p.Phe285Leu) mutations (25). Both had short stature (height SDS: -2.1 and -3.1$),$ IGF-1 deficiency ( -2.3 and -2.4), dysmorphic features and were SGA (birth weight SDS: -2.1 and -3.0). The phenotype of the parents of patient 41 is unknown and we do not have parental DNA. The mother of patient 42 has the same variant and a clinical phenotype of NS. Patient 43 had isolated short stature and a novel heterozygous c.3418T $>\mathrm{A}$ (p.Leu1140Ile) SOS1 variant predicted disease causing by Mutation taster (Table 2). This patient's father also has a similar phenotype of isolated short stature $(-2.4$ SDS) but parental DNA was not available to confirm the segregation.

\section{M syndrome genes}

Patients 44 and 45 had previously observed defects in CCDC8 (c.612dupG, p.Lys205Glufs*59) and CUL7 (c.2988G>A, p.Trp996*), respectively and had a classical GHI phenotype (Table 2).

\section{Analysis 2}

43 remaining patients (all probands; 38 with GHI and 5 with IGF-1 insensitivity) were screened for variants 
in 153 biological candidate growth genes associated with: syndromic growth disorders, skeletal dysplasias, growth plate biology, cell proliferation, DNA repair or growth retardation in mice (Supplementary Table 1). A homozygous variant was identified in one patient in FANCA (c.2000C>G, p.P667R; mother heterozygous, paternal DNA not available) predicted damaging by SIFT and probably damaging by PolyPhen. A homozygous variant was identified in one patient in $P H K B$ (c.56-1G>A; adopted child therefore parental DNA not available), which is associated with glycogen storage disease type IX (GSD IX). This alters one of the canonical splice site bases and is likely to cause exon skipping and an aberrant protein. 2 variants were identified in MDC1 (c.3774_3775delGCinsAT, p.P1259S and c.3528_3529delGCinsAT, p.P1177S; both predicted tolerated/benign by PolyPhen and SIFT) in one patient and 2 variants in another patient in EVC2 (c.673G>T, p.A225S ; and c.664T>A, p.F222I; possibly and probably deleterious by PolyPhen, respectively) which were inherited together in cis from one parent who has normal stature. The FANCA and $P H K B$ variants are potential candidates to explain the phenotype in 2 patients. In contrast, due to the in silico predictions and mode of inheritance respectively, the MDC1 and EVC2 variants were presumed non-pathogenic.

\section{Analysis 3}

In light of the dearth of variants identified by Analysis 2, WES data from all 43 undiagnosed patients were investigated using an unbiased approach. This strategy produced a shortlist of 109 variants in 77 candidate genes. Variants in all 77 genes were seen in GHI patients but only 4 genes had variants in patients with IGF-1 insensitivity (*in Supplementary Table 3), none of which were specific to IGF-1 insensitivity. PubMed and OMIM did not reveal obvious growth associations of the 77 candidate genes and pathway analysis did not reveal any enriched functional pathways. On rare variant burden testing, none of the 77 candidate genes were found to be significantly enriched for deleterious variants in cases vs controls. Therefore, the significance of these variants is uncertain.

\section{Associations between phenotypic features and genetic defects}

Although there was significant overlap, patients with identified genetic defects were significantly shorter compared to those with no genetic diagnosis (mean height SDS: -5.2 vs $-3.7 ; P<0.0001$ ) (Fig. 3). Height
SDS was significantly lower in patients with $G H R$ or $3 \mathrm{M}$ gene mutations compared to individuals with no genetic diagnosis (both $P<0.0001$ ) (Table 3). IGF-1 SDS values were significantly lower in patients with any genetic defect and in those with GHR mutations compared to individuals with no genetic diagnosis $(P=0.0128$ and $<0.0001$, respectively). GH levels were obtained from a number of different referral centres and likely measured by more than one assay. However, taking this limitation into account, peak GH levels were significantly higher in patients with GHR mutations compared to those with no genetic diagnosis $(P=0.0177)$ (Table 3$)$. Patients with $G H R$ $6 \Psi$ mutations had less severe phenotypes when compared to patients with other homozygous GHR defects (Mean height SDS -4.07 vs -6.2 respectively, $P=0.0006)$ as previously described (26). Consanguinity was predictive for identifying a molecular defect but age and sex were not (Supplementary Table 4).

\section{Discussion}

In approximately $80 \%$ of patients with short stature the aetiology remains elusive despite detailed clinical, biochemical and radiological assessment (27). This includes patients with extreme or syndromic short stature. Growth hormone (GHI) and IGF-1 insensitivity encompass a spectrum of clinical and biochemical abnormalities associated with normal GH secretion (1). The degree of short stature is variable in this group of disorders but in many cases the growth failure is severe.

The majority of referrals to our genetic sequencing service were male as previously described (10). Our cohort was heterogeneous but all patients had a phenotype consistent with GH or IGF-1 insensitivity i.e. short stature (height SDS $\leq-2.0$ ), GH sufficiency (peak GH $\geq 7.0 \mu \mathrm{g} / \mathrm{L}$ ) and low or normal/elevated IGF-1 levels, respectively.

Multiple mutations have been discovered in the GH-IGF-1 axis in association with GH and IGF-1 insensitivity including mutations in the GH1, GHR, STAT5B, IGFALS, PAPPA2, IGF1 and IGF1R genes $(1,7)$. We recently noted that, as well as the classically recognised GH-IGF-1 axis gene defects, other short stature disorders may have features of GHI such as 3M, Noonan and SilverRussell (SRS) syndromes (10). Consequently, we now routinely screen GHI patients born small for gestational age (SGA) for mutations in the $3 \mathrm{M}$ syndrome genes (OBSL1, CUL7 and CCDC8) as well as IGF1. Genetic testing for SRS was not carried out on other undiagnosed SGA subjects in this cohort; therefore, it is possible that 
Table 2 Clinical, biochemical and genetic features of patients diagnosed by whole-exome sequencing (WES) (total 11 patients, 12 variants).

\begin{tabular}{|c|c|c|c|c|c|c|c|c|c|c|c|}
\hline $\begin{array}{l}\text { Patient } \\
\text { number }\end{array}$ & Age (year) & Sex & $\begin{array}{l}\text { Consanguinity/ } \\
\text { ethnicity }\end{array}$ & $\begin{array}{c}\text { Birth } \\
\text { weight } \\
\text { SDS }\end{array}$ & $\begin{array}{l}\text { Height } \\
\text { SDS }\end{array}$ & $\begin{array}{l}\text { BMI } \\
\text { SDS }\end{array}$ & $\begin{array}{c}\text { Target } \\
\text { Height } \\
\text { SDS }\end{array}$ & $\begin{array}{c}\text { GH basal } \\
(\mu \mathrm{g} / \mathrm{L})\end{array}$ & $\begin{array}{c}\text { GH max } \\
(\mu \mathrm{g} / \mathrm{L})\end{array}$ & $\begin{array}{c}\text { IGF-1 } \\
\text { (ng/mL) }\end{array}$ & IGF-1 SDS \\
\hline 41 & 6.9 & $\mathrm{M}$ & +/Kuwaiti & 0.3 & -2.1 & -2.7 & -1.5 & 1.1 & $>32$ & 35.4 & -2.3 \\
\hline 42 & 8.9 & $\mathrm{~F}$ & -/Polish & -2.1 & -3.2 & -1.6 & 0.6 & 21.7 & 10.5 & 47 & -2.4 \\
\hline 43 & 13.1 & $\mathrm{M}$ & -/Mexican-Russian & -3.0 & -3.8 & -1.5 & -1.5 & 0.4 & 26.6 & 7.4 & -2.63 \\
\hline 44 & 1.9 & $\mathrm{~F}$ & +/Pakistani & -3.5 & -5.7 & -1.6 & $\mathrm{~N} / \mathrm{D}$ & 3.0 & 5.0 & 7.5 & -1.8 \\
\hline 45 & 0.3 & $\mathrm{~F}$ & +/Kuwaiti & -5.8 & -5.5 & -0.6 & NK & 22.5 & 26.7 & 116 & -1.1 \\
\hline 46 & 15.4 & $\mathrm{~F}$ & +/Pakistani & -3.4 & -5.0 & 1.0 & -3.4 & 0.1 & 13 & 35 & -2.5 \\
\hline $47^{\mathrm{h}}$ & 3.5 & $\mathrm{~F}$ & +/Kuwaiti & -0.82 & -3.9 & -1.2 & -1.8 & 9.5 & 85 & $<30$ & -5.3 \\
\hline $48^{h}$ & 2.2 & $F$ & +/Kuwaiti & -0.34 & -2.5 & -0.4 & 1.8 & 22 & ND & $<30$ & -5.3 \\
\hline 49 & 2.0 & $F$ & +/Kuwaiti & -2.1 & -6.7 & -2.3 & 0.3 & 35 & $>35$ & $<30$ & -5.3 \\
\hline 50 & 1.2 & $\mathrm{~F}$ & +/Kuwaiti & +0.12 & -6.7 & -1.3 & -1.4 & 11.3 & $>32$ & $<10$ & -2.1 \\
\hline 51 & 1.4 & $\mathrm{M}$ & +/Kuwaiti & +1.99 & -5.9 & -0.2 & -0.1 & $>35$ & $>35$ & $<30$ & -5.3 \\
\hline
\end{tabular}

other cases of SRS might have been missed. A proportion of short patients may carry disease-causing copy number variation (CNVs) or gene deletions/microdeletions (28) and analyses to detect this are currently underway in our undiagnosed patients. As such, deletions of candidate genes may have been missed by our analysis. Although detecting CNVs from WES is challenging, the use of algorithms may facilitate this process (29).

CGS using Sanger sequencing is based on the selection of appropriate gene(s) for analysis depending on the patient's clinical phenotype and hormonal profile. This approach is reliant on accurate clinical information available at the time of referral and is usually restricted to a small number of genes due to the time and cost implications. In contrast, next generation sequencing techniques such as targeted gene panels can be employed to analyse all genes known to cause a genetically heterogeneous disorder in one test. Alternatively, WES, allows the simultaneous analysis of all genes. Although gene panels can be powerful diagnostic tools, the advantage of WES is that data can also be mined for deleterious variants in novel genes not previously linked with a disease. Today, WES can be undertaken with a relatively low cost, however the interpretation of results can be difficult in inexperienced hands and the coverage of genes can be variable.

The traditional (CGS) approach alone confirmed a diagnosis in $35 \%$ of our cohort (31\% probands); the majority of cases (92\%) were diagnosed following 


\begin{tabular}{|c|c|c|c|c|c|c|c|c|}
\hline & & Genetic v & ariants & & & & & \\
\hline $\begin{array}{l}\text { Clinical } \\
\text { features }\end{array}$ & $\begin{array}{l}\text { Noonan } \\
\text { syndrome } \\
\text { genes }\end{array}$ & $\begin{array}{l}3 \mathrm{M} \text { syndrome } \\
\text { genes }\end{array}$ & IGFALS gene & GHR gene & MAFEXAC & $\begin{array}{l}\text { Predicted } \\
\text { outcome (novel } \\
\text { variants) }\end{array}$ & Reference & $\begin{array}{l}\text { No. genes } \\
\text { analysed } \\
\text { by CGS }\end{array}$ \\
\hline $\begin{array}{l}\text { ow set ears, } \\
\text { undescended } \\
\text { left testis }\end{array}$ & $\begin{array}{l}\text { PTPN11 Het } \\
\text { c.417G >C } \\
\text { p.Glu139Asp } \\
\text { (p.E139D) }\end{array}$ & & & & 0 & - & (25) & $1(G H R)$ \\
\hline $\begin{array}{l}\text {-ow set ears, } \\
\text { hypertelorism, } \\
\text { mild ptosis, } \\
\text { low posterior } \\
\text { hairline }\end{array}$ & $\begin{array}{l}\text { PTPN11 Het } \\
\text { c.853T>C, } \\
\text { p.Phe285Leu } \\
\text { (p.F285L) }\end{array}$ & & & & 0 & - & (25) & $1(G H R)$ \\
\hline No & $\begin{array}{l}\text { SOS1 Het } \\
\text { c.3418T>A } \\
\text { p.Leu1140Ile }\end{array}$ & & & & $3.5 \times E^{-5 x}$ & $\begin{array}{l}\text { Disease causing } \\
\text { (Mutation Taster) }\end{array}$ & Unpublished & $\begin{array}{l}2 \text { (GHR, } \\
\text { IGFALS) }\end{array}$ \\
\hline Classical & & $\begin{array}{l}C C D C 8 \text { Hom } \\
\text { c.612dupG, } \\
\text { p.Lys205Glufs*59 } \\
\text { (p.Lys205GlufsX59) }\end{array}$ & & & $1.8 \times \mathrm{E}^{-5 \mathrm{x}}$ & - & (23) & $1(G H R)$ \\
\hline $\begin{array}{l}\text { Classical } \\
\text { bilateral DDH }\end{array}$ & & $\begin{array}{l}\text { CUL7 Hom } \\
\text { c.2988G }>A \text {, } \\
\text { p.Trp996* } \\
\text { (pW996X) }\end{array}$ & & & 0 & - & (45) & $1(G H R)$ \\
\hline Classical & & & $\begin{array}{l}\text { c. } 1576 \mathrm{G}>\mathrm{A} \\
\text { p.Asp526Asn } \\
\text { c.632G }>\text { A, } \\
\text { p.Trp211* }\end{array}$ & & $\begin{array}{c}1.7 \times E^{-4 x} \\
0\end{array}$ & $\begin{array}{l}\text { Deleterious (SIFT } \\
\text { score 0) } \\
\text { Deleterious (SIFT } \\
\text { score 0) }\end{array}$ & $\begin{array}{l}\text { Unpublished } \\
\text { Unpublished }\end{array}$ & $1(G H R)$ \\
\hline Classical & & & & $\begin{array}{l}\text { Hom } \\
\text { c. } 70+4 A>C, \text { p.? }\end{array}$ & 0 & $\begin{array}{l}\text { Variation } \\
\text { predicted by HSF }\end{array}$ & Unpublished & $1(G H R)$ \\
\hline Classical & & & & $\begin{array}{l}\text { Hom } \\
\text { c. } 70+4 A>C, \text { p.? }\end{array}$ & 0 & $\begin{array}{l}\text { Variation } \\
\text { predicted by HSF }\end{array}$ & Unpublished & $1(G H R)$ \\
\hline Classical & & & & $\begin{array}{l}\text { Hom } \\
\text { c. } 70+4 A>C, \text { p.? }\end{array}$ & 0 & $\begin{array}{l}\text { Variation } \\
\text { predicted by HSF }\end{array}$ & Unpublished & $1(G H R)$ \\
\hline Classical & & & & $\begin{array}{l}\text { Hom } \\
\text { c. } 70+4 A>C, \text { p.? }\end{array}$ & 0 & $\begin{array}{l}\text { Variation } \\
\text { predicted by HSF }\end{array}$ & Unpublished & $1(G H R)$ \\
\hline Classical & & & & $\begin{array}{l}\text { Hom. c.703C>T, } \\
\text { p.Arg235* } \\
\text { (R217X) }\end{array}$ & $8.2 \times E^{-6 x}$ & - & (39) & $1(G H R)$ \\
\hline
\end{tabular}

Novel genetic variants are in bold font. Patients that underwent WES had no genetic diagnosis obtained following candidate gene sequencing. Height SDS is at presentation. ND, not done; +, parents consanguineous; -, parents not consanguineous; classical, classical GHI phenotype (frontal bossing, mid-facial hypoplasia); No, no dysmorphic features; DDH, developmental dysplasia of the hip; Hom, homozygous; Het, heterozygous; VEP, variant effect predictor; h, sisters. 3M syndrome genes, CUL7, CCDC8 and OBSL1. Noonan syndrome genes, PTPN11, SOS1. c. coding DNA sequence where nucleotide 1 is the A of the ATG-translation initiation codon, for GHR the transcript includes exon 3 NCBI Reference Sequences NM_000163; for IGFALS NM_004970; for CUL7 NM_014780; for CCDC8 NM_032040; for SOS1 NM_005633; PTPN11 NM_001330437; ins, insertion; fs, frameshift; *, termination site; as, acceptor site; ds, donor site; X, stop codon; del, deletion; ${ }^{\beta}$ predicted result if exon is skipped. c, coding DNA sequence where nucleotide 1 is the $A$ of the ATG-translation initiation codon of OBSL1 gene, NCBI reference NM_015311.2; MAF, minor allele frequency - variants are defined as rare if the MAF is $<0.001(0.1 \%)$ as recorded on the ExAC (Exome Aggregation Consortium) database; ${ }^{\mathrm{x}}$, no homozygotes in ExAC database. Variation predicted by HSF, HSF predicts exon skipping to be more likely than in reference allele; SIFT score 0 is deleterious, 1 is benign. References refer to the genetic variants. No. genes sequenced by CGS, number of genes (and which genes) sequenced by candidate gene sequencing (CGS) before proceeding to whole-exome sequencing (WES). Variant nomenclature is according to the HGVS guidelines.

sequencing of 1 or 2 genes. This technique is therefore relatively reliable if the phenotype is accurately documented and is typical for the disorder e.g. extreme short stature and IGF-1 deficiency (IGFD) with classical
Laron syndrome features (10, 30). Interestingly, we isolated a further 8 genetic variants in 11 patients in GHI genes by WES. These were not initially detected by CGS either because the variant was outside of the region 


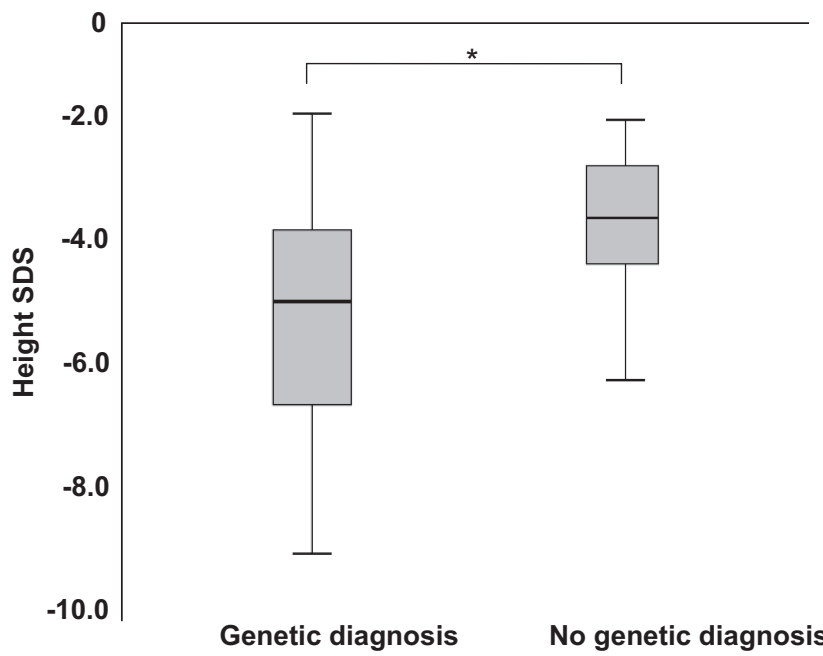

Figure 3

Height SDS in patients with a genetic diagnosis and those with no genetic diagnosis. Diagnosed patients $n=50$, undiagnosed $n=55 ;{ }^{\star} P=0.0001$.

amplified by Sanger sequencing in the case of the novel homozygous GHR gene mutation identified in 4 Kuwaiti patients or the phenotype was atypical (IGFALS, PTPN11, SOS1, CCDC8, CUL7). In the final Kuwaiti patient, the homozygous GHR mutation had been missed as a result of human error. Clinical phenotyping can be challenging for even experienced clinicians and many conditions have a wide phenotypic spectrum. In retrospect, the referring clinicians identified clinical features associated with Noonan and $3 \mathrm{M}$ syndromes in the 2 patients with previously reported PTPN11 mutations and the patients with CUL7 and OBSL1 mutations, respectively. The patient with a novel heterozygous SOS1 gene variant was born SGA, had short stature and IGFD but no classical features of NS. The other patient with novel compound heterozygous IGFALS gene variants is shorter (-5.0 SDS) than most/all previously reported patients with IGFALS defects (1). This emphasises not only the importance of accurate clinical phenotyping prior to referral for genetic testing but also the difficulties in diagnosing many short stature syndromes. Noonan in particular, should be carefully considered when assessing a patient with features of GHI (31).

Eleven novel genetic variants were identified in $G H R$, IGFALS and SOS1 genes. As functional studies were not undertaken on the novel variants, it remains a possibility that they are not responsible for the clinical phenotype. However, familial segregation and in silico prediction programmes have been utilised to substantiate them.

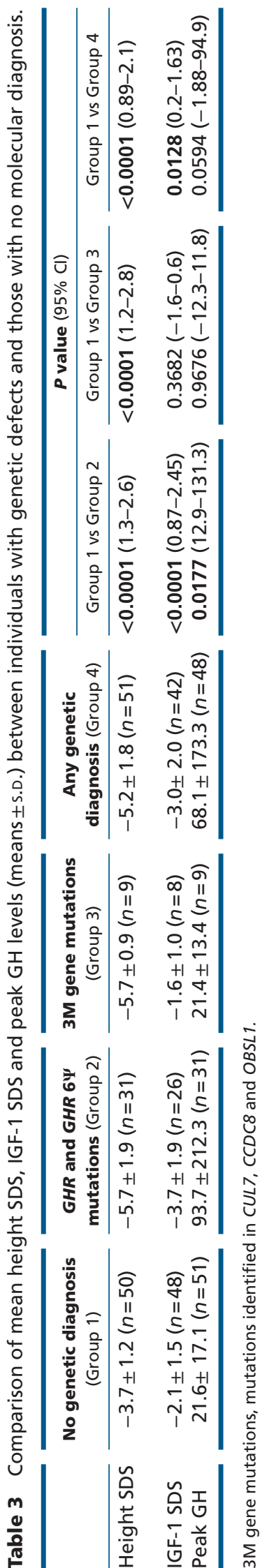


Except for cases 42 (compound heterozygous IGFALS) and 45 (heterozygous SOS1) the phenotypes are also typical for the identified genetic defects (1). Therefore, we are confident that these genetic variants explain the clinical presentation. According to ExAC, the SOS1 gene is intolerant of loss of function variants (expected number of loss of function variants 57.5; observed loss of function variants $3, \mathrm{pLI}=1.0$ ) this increases the likelihood of this variant being pathogenic. The IGFALS variants are both predicted to be highly deleterious and the patient had reduced birth weight (SDS -3.4). Together these factors may contribute to the development of a more extreme phenotype. No dysmorphic features or other potential genetic variants in candidate genes were identified in this patient that could explain the more severe phenotype. However, we cannot rule out oligogenicity with a novel gene defect. Prenatal growth retardation in particular has been previously recognised to contribute to the heterogeneity of IGFALS defects (32).

We also identified a novel homozygous, predicted deleterious FANCA mutation in a patient with normal birth weight, short stature (-3.0 SDS) and IGFD (-2.0). Fanconi anaemia (FA), is an autosomal recessive trait, associated with skeletal and cardiac defects, pre- and post-natal growth retardation and malformation of the kidneys, although consistent with this case, $25 \%$ patients have no reported physical abnormalities (33). The mean age at presentation is typical (usually $\sim 7$ years) and short stature is recognised presenting feature in children (33). Chromosome breakage test with mitomycin C (MMC) did not show any spontaneous chromosome fragility. Unfortunately, a lack of chromosomal fragility does not exclude FA and further investigations are currently underway. GSD IX is caused by PHKB mutations resulting in phosphorylase kinase deficiency. The novel, predicted damaging homozygous mutation was identified in a child with severe short stature ( -4.5 SDS) and IGFD ( -4.1 SDS). PHKB has an autosomal recessive mode of inheritance and the symptoms, severity and prognosis are highly variable, even among individuals with the same mutation. Characteristic features include, hepatomegaly, hypotonia, fasting hypoglycaemia and growth/pubertal delay. Although growth delays can be pronounced in affected children, catch-up growth is common and normal adult height is usually attained (34). This patient is under investigation by the local metabolic team.

The identification of FA and GSD in children is crucial to initiate close monitoring for serious long-term complications (haematological malignancies/hepatic and cardiac, respectively) and studies are underway to validate these diagnoses. Ideally, functional studies should be undertaken to definitively attribute the FANCA and $P H K B$ mutations to the phenotypes. Due to the in silico predictions and mode of inheritance respectively, the MDC1 and EVC2 variants were presumed non-pathogenic and have not been further investigated. The molecular diagnosis of all but 2 patients in the cohort could have potentially been secured using a next generation sequencing panel encompassing the genes included in Analysis 2. However, the advantage of WES is that it may serendipitously reveal a serious paediatric disorder, such as FA or GSD, which may have longer-term medical implications. Additionally, a gene panel would need to be continuously updated as further genetic causes of short stature are discovered. Furthermore, the cost of WES is significantly cheaper than undertaking CGS of the 22 genes known to cause GH and IGF-1 insensitivity (Analysis 1) i.e. approximately $£ 600$ vs $£ 1750$.

The identification novel genetic causes of short stature is essential to advance our understanding and management of growth disorders. To address this, we used an unbiased approach (Analysis 3) to uncover variants in genes, which might represent novel aetiologies for short stature. No strong candidate gene(s) emerged from this analysis, but we hereby report the results for reference. The failure to identify other genes may be a result of wider genetic heterogeneity i.e. numerous other undiscovered growth genes exist which have not been identified in our relatively small cohort. Oligogenic inheritance of genes known to cause short stature may also explain some short stature phenotypes, although Analysis 2 does not support this. It is also possible that a combination of both these factors may be important. As we were unable to perform trio analysis on all patients, we may have missed some de novo variants acting in a dominant fashion. Additionally, $\mathrm{CNV}$ or unexplored e.g. intronic or regulatory regions of the known genes not covered by WES, such as the GHR pseudoexon mutation, may contribute $(26,28)$. In the coming years, whole genome sequencing will uncover more such examples.

Deciding which short patients to refer for genetic testing can be problematic. Knowledge of the clinical features associated with different gene mutations is key to deciding which gene to prioritise. Our data suggest that accurate assessment of height, IGF-1 and GH may improve the diagnostic yield. Additionally, a genetic defect is more likely to be identified in consanguineous offspring. The current study suggests that CGS is reliable when the clinical features and the biochemical profile 
strongly suggest a particular candidate gene e.g. a GHR mutation. However, if a genetic diagnosis is not secured following sequencing of two candidate genes, then the CGS strategy is unlikely to reveal a genetic diagnosis, and it is also more cost effective to proceed to WES.

We present the results of comprehensive genetic testing in a cohort of patients with GH and IGF-1 insensitivity. A number of novel defects were identified in several genes associated with GH and IGF insensitivity. Our data expand the phenotypes associated with several genetic defects and also the spectrum of overlapping diagnoses associated with GHI. Next-generation sequencing is an important adjuvant to CGS in the diagnosis of genetic short stature and emphasises the benefit of specialist diagnostic centres.

\section{Supplementary data}

This is linked to the online version of the paper at http://dx.doi.org/10.1530/ EJE-17-0453.

\section{Declaration of interest}

H S has received honoraria from Ipsen Pharmaceuticals and research grants from Ipsen and Sandoz Pharmaceuticals. MOS has a consultancy agreement with Ipsen Pharmaceuticals.

\section{Funding}

The genetic sequencing service was supported by a research grant from Ipsen UK (H L S). L S was supported by a Sandoz Pharmaceutical sponsored Clinical Training Fellowship.

\section{Author contribution statement}

L A M and L S performed bioinformatics analyses. S C, D G R, K M D and $\mathrm{H}$ L S contributed to patient recruitment, data collection and analysis. S C performed the phenotypic and statistical analyses. H L S wrote the manuscript with input from S C, L A M, L S and D G R.

\section{References}

1 David A, Hwa V, Metherell LA, Netchine I, Camacho-Hubner C, Clark AJ, Rosenfeld RG \& Savage MO. Evidence for a continuum of genetic, phenotypic, and biochemical abnormalities in children with growth hormone insensitivity. Endocrine Reviews 201132 472-497. (doi:10.1210/er.2010-0023)

2 Godowski PJ, Leung DW, Meacham LR, Galgani JP, Hellmiss R, Keret R, Rotwein PS, Parks JS, Laron Z \& Wood WI. Characterization of the human growth hormone receptor gene and demonstration of a partial gene deletion in two patients with Laron-type dwarfism. PNAS 198986 8083-8087. (doi:10.1073/pnas.86.20.8083)

3 Amselem S, Duquesnoy P, Attree O, Novelli G, Bousnina S, PostelVinay MC \& Goossens M. Laron dwarfism and mutations of the growth hormone-receptor gene. New England Journal of Medicine 1989 321 989-995. (doi:10.1056/NEJM198910123211501)
4 Kofoed EM, Hwa V, Little B, Woods KA, Buckway CK, Tsubaki J, Pratt KL, Bezrodnik L, Jasper H, Tepper A et al. Growth hormone insensitivity associated with a STAT5b mutation. New England Journal of Medicine 2003349 1139-1147. (doi:10.1056/NEJMoa022926)

5 Rosenfeld RG, Belgorosky A, Camacho-Hubner C, Savage MO, Wit JM \& Hwa V. Defects in growth hormone receptor signaling. Trends in Endocrinology and Metabolism: TEM 200718 134-141. (doi:10.1016/j. tem.2007.03.004)

6 Domene HM, Bengolea SV, Martinez AS, Ropelato MG, Pennisi P, Scaglia P, Heinrich JJ \& Jasper HG. Deficiency of the circulating insulin-like growth factor system associated with inactivation of the acid-labile subunit gene. New England Journal of Medicine $2004 \mathbf{3 5 0}$ 570-577. (doi:10.1056/NEJMoa013100)

7 Dauber A, Munoz-Calvo MT, Barrios V, Domene HM, Kloverpris S, Serra-Juhe C, Desikan V, Pozo J, Muzumdar R, Martos-Moreno GA et al. Mutations in pregnancy-associated plasma protein A2 cause short stature due to low IGF-I availability. EMBO Molecular Medicine 20168 363-374. (doi:10.15252/emmm.201506106)

8 Woods KA, Camacho-Hubner C, Savage MO \& Clark AJ. Intrauterine growth retardation and postnatal growth failure associated with deletion of the insulin-like growth factor I gene. New England Journal of Medicine 1996335 1363-1367. (doi:10.1056/ NEJM199610313351805)

9 Abuzzahab MJ, Schneider A, Goddard A, Grigorescu F, Lautier C, Keller E, Kiess W, Klammt J, Kratzsch J, Osgood D et al. IGF-I receptor mutations resulting in intrauterine and postnatal growth retardation. New England Journal of Medicine 2003349 2211-2222.

10 Storr HL, Dunkel L, Kowalczyk J, Savage MO \& Metherell LA. Genetic characterisation of a cohort of children clinically labelled as $\mathrm{GH}$ or IGF1 insensitive: diagnostic value of serum IGF1 and height at presentation. European Journal of Endocrinology 2015172 151-161. (doi:10.1530/EJE-14-0541)

11 Laron Z. Laron syndrome (primary growth hormone resistance or insensitivity): the personal experience 1958-2003. Journal of Clinical Endocrinology and Metabolism 200489 1031-1044. (doi:10.1210/ jc.2003-031033)

12 Domene HM, Hwa V, Argente J, Wit JM, Camacho-Hubner C, Jasper HG, Pozo J, van Duyvenvoorde HA, Yakar S, Fofanova-Gambetti $\mathrm{OV}$ et al. Human acid-labile subunit deficiency: clinical, endocrine and metabolic consequences. Hormone Research 200972 129-141. (doi:10.1159/000232486)

13 Akawi NA, Ali BR, Hamamy H, Al-Hadidy A \& Al-Gazali L. Is autosomal recessive Silver-Russel syndrome a separate entity or is it part of the 3-M syndrome spectrum? American Journal of Medical Genetics Part A 2011 155A 1236-1245. (doi:10.1002/ajmg.a.34009)

14 Binder G, Neuer K, Ranke MB \& Wittekindt NE. PTPN11 mutation are associated with mild growth hormone resistance in individuals with Noonan syndrome. Journal of Clinical Endocrinology and Metabolism 200590 5377-5381. (doi:10.1210/jc.2005-0995)

15 Miller JD, McKusick VA, Malvaux P, Temtamy S \& Salinas C. The 3-M syndrome: a heritable low birthweight dwarfism. Birth Defects Original Article Series 197511 39-47.

16 Hanson D, Murray PG, Black GC \& Clayton PE. The genetics of 3-M syndrome: unravelling a potential new regulatory growth pathway. Hormone Research in Paediatrics 201176 369-378. (doi:10.1159/000334392)

17 Hanson D, Murray PG, Coulson T, Sud A, Omokanye A, Stratta E, Sakhinia F, Bonshek C, Wilson LC, Wakeling E et al. Mutations in CUL7, OBSL1 and CCDC8 in 3-M syndrome lead to disordered growth factor signalling. Journal of Molecular Endocrinology 201249 267-275. (doi:10.1530/JME-12-0034)

18 Abu-Amero S, Monk D, Frost J, Preece M, Stanier P \& Moore GE. The genetic aetiology of Silver-Russell syndrome. Journal of Medical Genetics 200845 193-199. (doi:10.1136/jmg.2007.053017)

19 Yamamoto GL, Aguena M, Gos M, Hung C, Pilch J, Fahiminiya S, Abramowicz A, Cristian I, Buscarilli M, Naslavsky MS et al. Rare 
variants in SOS2 and LZTR1 are associated with Noonan syndrome. Journal of Medical Genetics 201552 413-421. (doi:10.1136/ jmedgenet-2015-103018)

20 Tafazoli A, Eshraghi P, Koleti ZK \& Abbaszadegan M. Noonan syndrome-a new survey. Archives of Medical Science 201713 215-222. (doi:10.5114/aoms.2017.64720)

21 Chernausek SD, Backeljauw PF, Frane J, Kuntze J \& Underwood LE. Long-term treatment with recombinant insulin-like growth factor (IGF)-I in children with severe IGF-I deficiency due to growth hormone insensitivity. The Journal of Clinical Endocrinology and Metabolism 200792 902-910. (doi:10.1210/jc.2006-1610)

22 Chan LF, Campbell DC, Novoselova TV, Clark AJ \& Metherell LA. Whole-exome sequencing in the differential diagnosis of primary adrenal insufficiency in children. Frontiers in Endocrinology 20156113.

23 Hanson D, Murray PG, Sud A, Temtamy SA, Aglan M, Superti-Furga A, Holder SE, Urquhart J, Hilton E, Manson FD et al. The primordial growth disorder 3-M syndrome connects ubiquitination to the cytoskeletal adaptor OBSL1. American Journal of Human Genetics 2009 84 801-806. (doi:10.1016/j.ajhg.2009.04.021)

24 Huber C, Dias-Santagata D, Glaser A, O'Sullivan J, Brauner R, Wu K, $\mathrm{Xu} \mathrm{X}$, Pearce K, Wang R, Uzielli ML et al. Identification of mutations in CUL7 in 3-M syndrome. Nature Genetics 200537 1119-1124. (doi:10.1038/ng1628)

25 Tartaglia M, Kalidas K, Shaw A, Song X, Musat DL, van der Burgt I, Brunner HG, Bertola DR, Crosby A, Ion A et al. PTPN11 mutations in Noonan syndrome: molecular spectrum, genotype-phenotype correlation, and phenotypic heterogeneity. American Journal of Human Genetics 200270 1555-1563. (doi:10.1086/340847)

26 Metherell LA, Akker SA, Munroe PB, Rose SJ, Caulfield M, Savage MO, Chew SL \& Clark AJ. Pseudoexon activation as a novel mechanism for disease resulting in atypical growth-hormone insensitivity. American Journal of Human Genetics 200169 641-646. (doi:10.1086/323266)

27 Cohen P, Rogol AD, Deal CL, Saenger P, Reiter EO, Ross JL, Chernausek SD, Savage MO \& Wit JM. Consensus statement on the diagnosis and treatment of children with idiopathic short stature: a summary of the Growth Hormone Research Society, the Lawson Wilkins Pediatric Endocrine Society, and the European Society for Paediatric Endocrinology Workshop. Journal of Clinical Endocrinology and Metabolism 200893 4210-4217. (doi:10.1210/jc.2008-0509)

28 Wit JM, Oostdijk W, Losekoot M, van Duyvenvoorde HA, Ruivenkamp CA \& Kant SG. Mechanisms in endocrinology: novel genetic causes of short stature. European Journal of Endocrinology 2016 174 R145-R173. (doi:10.1530/EJE-15-0937)

29 Fromer M, Moran JL, Chambert K, Banks E, Bergen SE, Ruderfer DM, Handsaker RE, McCarroll SA, O'Donovan MC, Owen MJ et al. Discovery and statistical genotyping of copy-number variation from whole-exome sequencing depth. American Journal of Human Genetics 201291 597-607. (doi:10.1016/j.ajhg.2012.08.005)

30 Wit JM, van Duyvenvoorde HA, Scheltinga SA, de Bruin S, Hafkenscheid L, Kant SG, Ruivenkamp CA, Gijsbers AC, van Doorn J, Feigerlova E et al. Genetic analysis of short children with apparent growth hormone insensitivity. Hormone Research in Paediatrics 2012 77 320-333. (doi:10.1159/000338462)

31 Romano AA, Allanson JE, Dahlgren J, Gelb BD, Hall B, Pierpont ME, Roberts AE, Robinson W, Takemoto CM \& Noonan JA. Noonan syndrome: clinical features, diagnosis, and management guidelines. Pediatrics 2010126 746-759. (doi:10.1542/peds.2009-3207)

32 Storr HL, Prasad R, Temple IK, Metherell LA, Savage MO \& Walker JM. Heterogeneity of the growth phenotype and birth size in acid-labile subunit (ALS) deficiency. Journal of Endocrinological Investigation 2015 38 407-412. (doi:10.1007/s40618-014-0195-1)

33 Alter BP. Diagnosis, genetics, and management of inherited bone marrow failure syndromes. Hematology: American Society of Hematology. Education Program 20071 29-39.

34 Roscher A, Patel J, Hewson S, Nagy L, Feigenbaum A, Kronick J, Raiman J, Schulze A, Siriwardena K \& Mercimek-Mahmutoglu S. The natural history of glycogen storage disease types VI and IX: long-term outcome from the largest metabolic center in Canada. Molecular Genetics and Metabolism 2014113 171-176. (doi:10.1016/j.ymgme.2014.09.005)

35 Khan YN, Raza J, Derr MA, Hwa V \& Rosenfeld RG. Identification of a novel mutation (L229P) in the GHR gene in a cohort of Pakistani patients with growth hormone insensitivity and severe growth retardation. Hormone Research 200972.

36 Berg MA, Guevara-Aguirre J, Rosenbloom AL, Rosenfeld RG \& Francke U. Mutation creating a new splice site in the growth hormone receptor genes of 37 Ecuadorean patients with Laron syndrome. Human Mutation 19921 24-32. (doi:10.1002/humu.1380010105)

37 David A, Miraki-Moud F, Shaw NJ, Savage MO, Clark AJ \& Metherell LA. Identification and characterisation of a novel GHR defect disrupting the polypyrimidine tract and resulting in $\mathrm{GH}$ insensitivity. European Journal of Endocrinology 2010162 37-42. (doi:10.1530/EJE-09-0583)

38 Sobrier ML, Dastot F, Duquesnoy P, Kandemir N, Yordam N, Goossens $\mathrm{M} \&$ Amselem S. Nine novel growth hormone receptor gene mutations in patients with Laron syndrome. Journal of Clinical Endocrinology and Metabolism 199782 435-437.

39 Amselem S, Duquesnoy P, Duriez B, Dastot F, Sobrier ML, Valleix S \& Goossens M. Spectrum of growth hormone receptor mutations and associated haplotypes in Laron syndrome. Human Molecular Genetics 19932 355-359. (doi:10.1093/hmg/2.4.355)

40 Feigerlova E, Swinyard M, Derr MA, Farnsworth J, Andrew SF, Rosenfeld RG \& Hwa V. A novel GHR intronic variant, c. $266+83 \mathrm{G}>\mathrm{T}$, activates a cryptic 5 ' splice site causing severe GHR deficiency and classical GH insensitivity syndrome. Hormone Research in Paediatrics 201380 397-405. (doi:10.1159/000355404)

41 Baumbach L, Schiavi A, Bartlett R, Perera E, Day J, Brown MR, Stein S, Eidson M, Parks JS \& Cleveland W. Clinical biochemical, and molecular investigations of a genetic isolate of growth hormone insensitivity (Laron's syndrome). Journal of Clinical Endocrinology and Metabolism 199782 444-451.

42 Amselem S, Sobrier ML, Duquesnoy P, Rappaport R, Postel-Vinay MC, Gourmelen M, Dallapiccola B \& Goossens M. Recurrent nonsense mutations in the growth hormone receptor from patients with Laron dwarfism. Journal of Clinical Investigation 199187 1098-1102. (doi:10.1172/JCI115071)

43 David A, Rose SJ, Miraki-Moud F, Metherell LA, Savage MO, Clark AJ \& Camacho-Hubner C. Acid-labile subunit deficiency and growth failure: description of two novel cases. Hormone Research in Paediatrics 201073 328-334. (doi:10.1159/000308164)

44 Huber C, Fradin M, Edouard T, Le Merrer M, Alanay Y, Da Silva DB, David A, Hamamy H, van Hest L, Lund AM, et al. OBSL1 mutations in 3-M syndrome are associated with a modulation of IGFBP2 and IGFBP5 expression levels. Human Mutation 201031 20-26. (doi:10.1002/humu.21150)

45 Al-Dosari MS, Al-Shammari M, Shaheen R, Faqeih E, Alghofely MA, Boukai A \& Alkuraya FS. 3M syndrome: an easily recognizable yet underdiagnosed cause of proportionate short stature. Journal of Pediatrics 2012161 139.e131-145.e131.
Received 5 June 2017

Revised version received 29 August 2017

Accepted 4 September 2017 